Note: This is a draft of a paper being submitted for publication. Contents of this paper should not be quoted nor referred to without permission of the author(s).

[To be submitted to the Symposium on Ion Beam Processing of Advanced Elecironic Materials, 1989 Spring Meeting of the Materials Research Society, San Diego, CA, April 24-29, 1989]

CONF-890426--24

DE89 015044

\title{
DAMAGE GROWTH IN Si DURING SELF-ION IRRADIATION: A STUDY OF ION EFFECTS OVER AN EXTENDED ENERGY RANGE
}

\author{
O. W. Holland, M. K. El-Ghor, and C. W. White
}

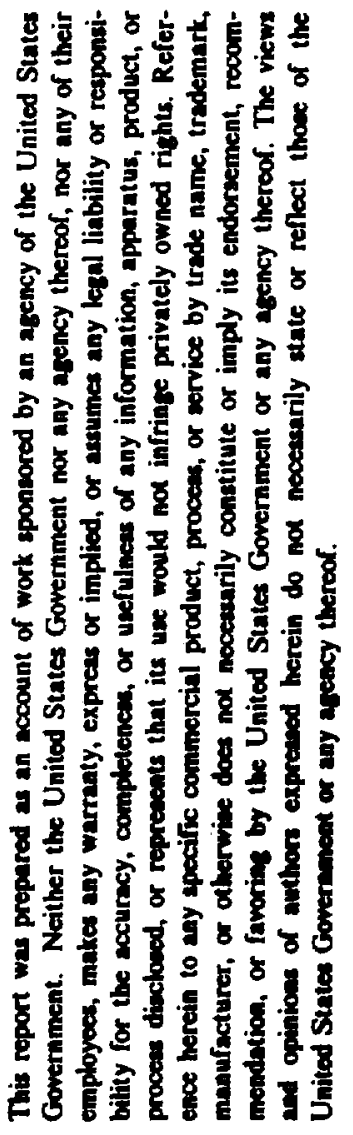

\begin{abstract}
"The submitted manuscript has been authored by a contractor of the U.S. Govemment under contract No. DEAC05-84OR21400. Accordingly, the U.S. Government retains a nonexclusive, rayalty-free license to publish or reproduce the published form of this contribution, or allow others to do so, for U.S. Government purposes."
\end{abstract}

Prepared by the

Solid State Division

Oak Ridge National Laboratory

Oak Ridge, Tennessee 37831

operated by

MARTIN MARIETTA ENERGY SYSTEMS, INC. for the

U.S. DEPARTMENT OF ENERGY

under contract DE-AC05-84OR21400 
(). HOH AND, M. K. RL-GHOR, AND C. W. WHITE, Solid State Division, Oak Ridge Vianonai Laboratory, Oak Ridge, TN 37831

\section{LBSTRACT}

Damage nucleation/growth in single-crystal Si during ion irradiation is discussed. For MeV inns, the rate of growth as was well as the damage morphology are shown to vary widely along the track of the ion. This is attributed to a change in the dominant, defect-related reactions as the ion penetrates the crystal. The nature of these reactions were elucidated by studying the interacion of $\mathrm{MeV}$ ions with different types of defects. The defects were introduced into the Si crystal prior to high-energy irradiation by self-ion implantation at a medium energy $(100 \mathrm{keV})$. Varied damage morphologies were produced by implanting different ion fluences. Electron microscopy and ion-channeling measurements, in conjunction with annealing studies, were used to characterize the damage. Subtle changes in the predamage morphology are shown to result in markedly difient responses to the high-energy imadiation, ranging from complete annealing of the damage to rapid growth. These divergent responses occur over a narrow range of dose $\left(2-3 \times 10^{14}\right.$ $\mathrm{cm}^{-2}$ ) of the nedium-energy ions; this range also marks a transition in the growth behavior of the camage during the predamaging implantation. A model is proposed which accounts for these observations and provides insight into ion-induced growth of amorphous layers in Si and the role of ite amorphous/crystalline interface in this process.

\section{INTRODLCTION}

The mecharisms responsible for damage accumulation in Si during heavy ion irradiation have been the subject of many investigations. Obviously many of the studies were motivated by the importailce of ion-related technologies in the manufacture of Si-integrated circuits. Also, the commercial availability of low-defect density, high-purity Si single crystal makes it an attractive material for studying such phenomena. While much progress has been made, no comprehensive model exists which can predict the nature of the ion-solid interaction over an extended range of ion malss and energy and substrate temperature. Part of the problem has been in identification of the many different defects which form during ion irradiation. The Frenkel defect (vacancyinterstitial pair), which is produced directly when ion scattering in the lattice results in atomic dispiacements, is mobile at RT (and bclow). These point-defects will participate in the formation of other defects; ones which are stable at the irradiation temperature. While much progress has been made in defect classification [1], little is known about their mutual interaction (which obviously plays a role in the evolution of the damage morphology during irradiation) or their formation kinetics.

The understanding of ion-related damage processes is further complicated by an uncertainty in the role of the collision cascade. The passage of an energetic ion through a solid will initiate a sequence of displacement events. If atoms are displaced with sufficient energy, their interaction with other lattice atoms can lead to further displacements. Frenkel pair production is therefore a cascide process in which the incident ion is simply the primary damage-producing particle. The total of all such events is commonly referred to as the collision cascade of the ion. One model envisions that stable damage is nucleated within these dense cascades during the time that it takes For the energy spike to dissipate $[2,3]$ (i.e., the time for cascade quenching, which is of the order of $10^{-12}$ ). Such a model has been referred to as heterogeneous because the nucleation of damage is localized in the vicinity of the collision cascade. An alternate model considers damage nucleation and growth to occur homogeneously within the lattice as a result of randon interaction between the point defects generated by different collision cascade $14 \mid$. Obviously in such a meciel, the point defects are assumed to be long-lived relative to the quench time of the cascade so that they become homogeneously distributed throughout the irradiated region without any spatial correlation to the cascade volume.

In this paper, damage nucleation and growth in single-crystal Si during self-ion irradiation over a wide range of ion energy is discussed. MeV ions were used because they offer the 
possibility of studying ion-impact phenomena over an extended range of nuclear and electronic stopping. The mature of the damage growth varied over the penetration depth of the high-energy ions. Two distinct regions were identified, and the growth behavior in each is discussed. Insight into the mechanisms responsible for the different behaviors was gained from studying the inferaction of the $\mathrm{MeV}$ ions with preexisting damage in the Si lattice. Predamaging of the lattice, F.: Ir to the $\Lambda \mathrm{eV}$-ion imadiation, was accomplished by $100-\mathrm{keV}$ self-ion irradiation. Different fluences were used to vary the predamage morphology. Characterization of the damage at the lower energy was done to better understand its interaction with the MeV ions. However, this characterization yielded interesting results which, when correlated with the higher-energy results, leads to a better understanding of ion-induced amorphization of Si and the role of the amorphous/ crystal interiace in this process. Analytical techniques used in the study included Rutherford backscattering/channeling spectroscopy (RBS) and cross-sectional transmission electron microscopy (TEM).

\section{EXPERIMENTAL}

Czochralski-grown, p-type Si (100) wafers with resistivities between 6-8 $\Omega$-cm were used in this study. High-energy implantations $(>1.0 \mathrm{MeV})$ were done with a raster-scanned beam of $28 \mathrm{Si}^{+}$-ions from a 1.7 MeV General Ionex tandem accelerator. An energy of $1.25 \mathrm{MeV}$ and an average current density $<0.4 \mu \mathrm{A} / \mathrm{cm}^{2}$ were generally used. The use of a tandem accelerator insures that the ${ }^{28} \mathrm{Si}^{+}$beam will be free of any contaminants, such as molecular ions of the same mass-to-charge ratio, as might be encountered using a single-ended machine. For lower energy implantations of the $\mathrm{Si}^{+}$self-ion (which were done with a single-ended, mass-analyzed implanter), isotope ${ }^{30} \mathrm{Si}$ was used to minimize the possibility of contaminant beams. Postimplantation annealing was carried out in a conventional, quartz-tube furnace in flowing, dry nitrogen gas. Structural characterization of cross-sectionally thinned samples was done using a Philips EM400T transmission electron microscopy. RBS with $2.75-\mathrm{MeV}-\mathrm{He}^{+}$ions was used to measure damage profiles.

\section{HISTORICAL PERSPECTIVE}

\section{Heterogeneous Model}

Brinkman [5] argued that, when the nuclear stopping of an ion is sufficiently high to displace at least one atom per lattice plane, there will be a net outward motion of the target atoms along the incident ion trajectory. Such correlated motion will produce a vacancy-rich core surrounded by an interstitial-rich region and is known as a displacement spike. He suggested that nucleation of extended defects such as dislocations can result from such a process. It is clear that the high concentration of defects localized within dense collision cascades offer other possibilities for damage nucleation. Swanson [6] studied the ion-induced, crystalline-to-amorphous (c-a) transformation in Si and concluded that it occurs spontaneously when the free energy of the defective crystalline regions becomes equal to or greater than that of the amorphous phase. If the defect density (deposited energy density) is sufficiently high then it was suggested that the c-a transformation can occur directly within the volume of the collision cascade. For less-dense cascades, it was envisioned that overlap of the damage regions produced by different ions was needed to achieve the critical defect density to stimulate the phase change. Discrete damaged regions associated with collision cascades of ions have been observed by TEM [7-9]. Figure 1(a) shows the cascade damage observed by plan-view TEM in (110) Si after implantation at $80 \mathrm{~K}$ with $100-\mathrm{keV} \mathrm{Bi}$ ions to a dose of $1.0 \times 10^{12} \mathrm{~cm}^{-2}$. The cascades appear as black/white spots with an average size of $50 \AA$. The image reversal in the figure occurs across a thickness contour in the sample. The absence of $<110>$ chains of atoms in the central region of the cascade damage is evident in the high-resolution micrograph in Fig. 1(b). Howe et al. 17] showed that the efficiency of creating such damaged regions was $<0.1$ when the deposited energy density was $<0.1 \mathrm{eV} /$ atom and was $>0.7$ when the density exceeded $0.4 \mathrm{eV} /$ atom. It is tempting to conclude from such studies that the formation of a continuous amorphous layer proceeds by a coalescence or overlapping of such regions. Gibbons [10] incorporated this idea into a model which allowed 
for amominzation to occur either within a single cascade or as a result of cascade overlap. In the model, the fractional amorphous area is given as

$$
\frac{A_{\Lambda}}{A_{0}}=1-\left(\sum_{k=0}^{m-1} \frac{\left(A_{j} \Phi\right)^{k}}{k !} \exp \left(-A_{i} \Phi\right)\right)
$$

where $m$ is the number of ions required to damage a region sufficiently to form the amorphous phase, $\Phi$ is the ion fluence, and $A_{i}$ is the cross-sectional area of the amorphous region formed around the track of the ion. Somewhat surprising, Dennis and Hale [11] found that, for a wide range of ions (from $\mathrm{Li}^{+}$to $\mathrm{Kr}^{+}$) and implantation temperature $(80-300 \mathrm{~K}$ ), the $\mathrm{m}$ value determined from fitting their data to Eqn. (1) was always greater than 1 and was within the 2-3 range. This is suggestive that some other mechanism for amorphization other than heterogeneous growth must be considered. This was further confirmed by the work of Ruault et al. [9] who observed very dense cascades in Si by TEM. The cascades were found to have an amorphous core, and their number density correlated well with the implanted fluence. However, it was shown that this type of damage could not account for the formation of the continuous amorphous layer observed at higher fluences. Rather, it was found that nucleation of amorphous Si occurred randomly in the regions between the damaged regions of the collision cascade. This growth mechanism appeared to be more dominant than the cascade mechanism, and produced an amorphous phase which had recrystallization kinetics similar to that of a continuous amorphous layer, while the amorphous cascade regions annealed at anomalously low temperatures.
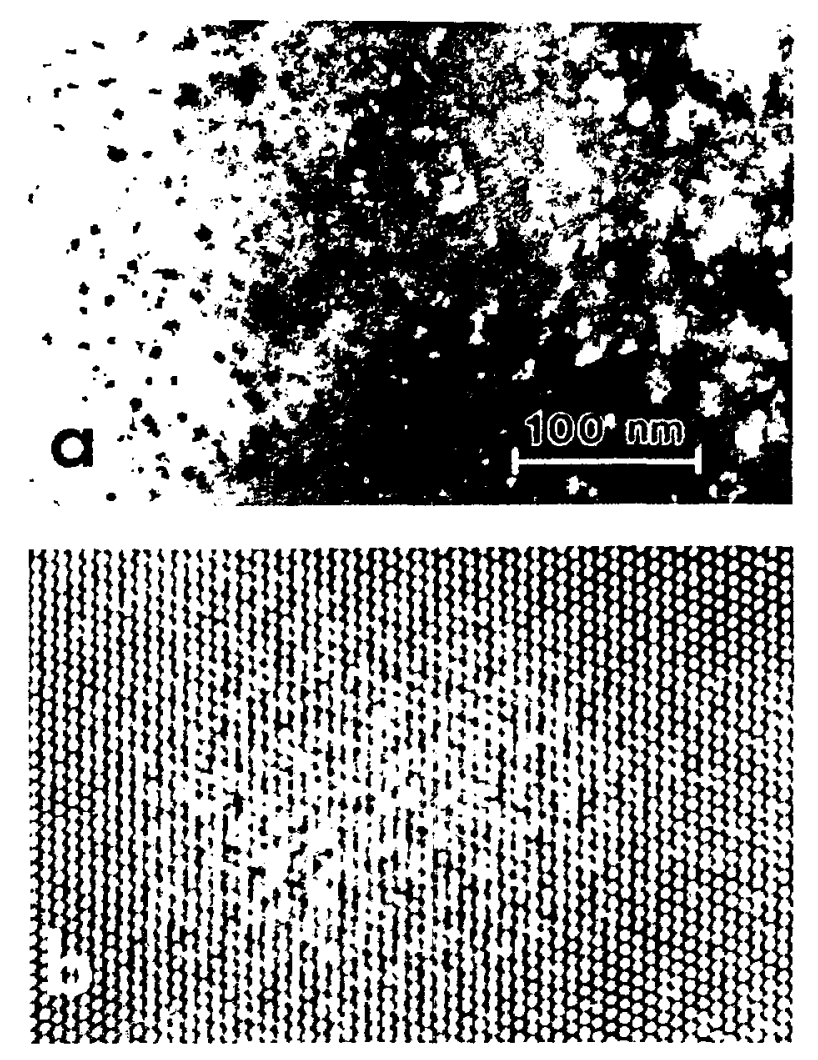

Fig. 1. (a) Bright-field electron micrograph in plan view of discrete damage produced by $\mathrm{Bi}^{+}$ $\left(1.0 \times 10^{12} \mathrm{~cm}^{-2}, 100 \mathrm{keV}\right.$, at $\left.\mathrm{LN}_{2}\right)$ implantation of $\mathrm{Si}(110)$, and (b) high-resolution image from a damaged region showing the amorphous structure.

\section{Homogeneous Model}

In contrast to the heterogeneous model, is the homogeneous model in which damage accumulation is envisioned to occur as a result of outdiffusion of the Frenkel defects from the cascade volume where they interact with defects from other cascades to nucleate stable complexes. Damage is therefore nucleated homogeneously, rather than locally within the region of 
the cuscade. Clearly, this model assumes that Frenkel defects formed within an ion cascade are long-lived so that any spatial correlation with the cascade volume is lost prior to their interaction. While it is reasonable to assume that this model is more appropriate to those cases where the ionsolid combination results in low-density cascades, the observations discussed above for dense cascades suggest that the homogeneous mechanism may be important over a larger range. While this model differs from the heterogencous one, amorphization of the lattice during irradiation is still thought to oceur by a spontaneous phase transformation when the local defect density exceeds the critical value. However, the onset of amorphization is expected to occur only after an incubation period (during which damage levels increase to the critical concentration), and thereafter the growth will occur abruptly over a narrow range in dose. This is in sharp contrast to the heterogeneous model [given in Eqn. (1)] and therefore provides a stringent test to differentiate between the two models.

\section{RESULTS}

\section{$1.25 \mathrm{MeV} \mathrm{Si}+$ Ion Irradiation}

Aligned and random spectra from Si after implantation with $1.25 \mathrm{Si}^{+}$ions are shown in Fig. 2. Spectra from two samples which had been implanted with $0.25 \times 10^{16}$ and $1.0 \times 10^{16}$ $\mathrm{cm}^{-2}$, respectively, are compared. The deviation of the aligned scattering yield at any point within the inplanted samples from that in the virgin sample indicates the presence of ion-induced damage. In the spectrum from the sample implanted with the high-dose (HD), the scattering yield is seen to be at the random level in a region centered on the ions' end of range, EOR, $(\sim 1.3$ $\mu \mathrm{m})$. This is consistent with the formation of a buried amorphous layer. By comparison, the amount of damage ahead of the EOR and extending to the surface is seen to be much reduced. The results of computer simulation using TRIM [12] of $1.25-\mathrm{MeV} \mathrm{Si}^{+}$ions in Si, normalized to an implant fluence of $1 \times 10^{15} \mathrm{~cm}^{-2}$, are shown in Fig. 3. The density of Frenkel defects

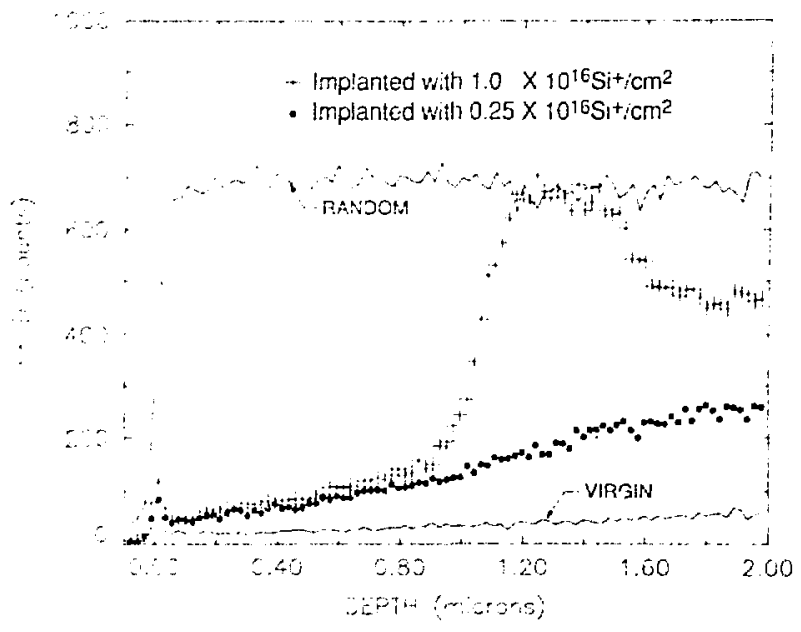

Fig. 2. $<100>$ aligned spectra from Si single crystals implanted with $1.25-\mathrm{MeV} \mathrm{Si}^{+}$ions.

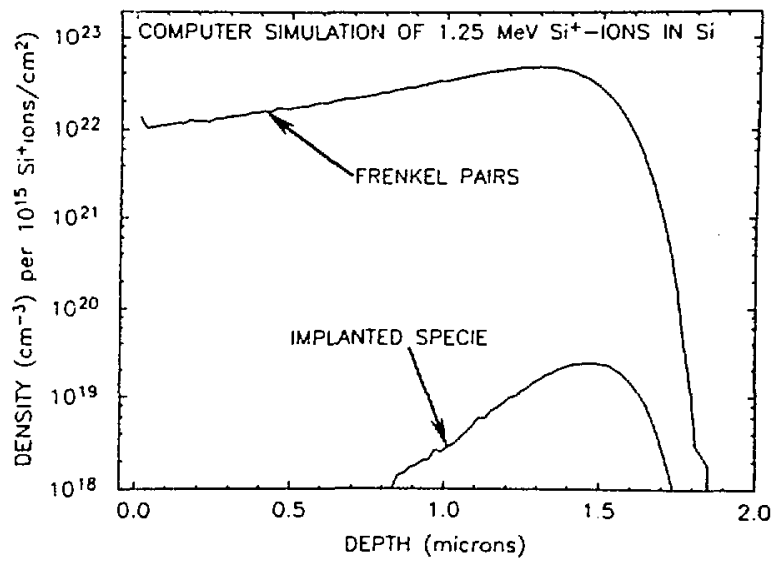

Fig. 3. TRIM calculation of the distribution of Frenkel defects and the implanted specie for 1.25-MeV Si+ ions in $\mathrm{Si}$.

produced along the path of the ion is seen to increase near the ions' EOR, but the variation is small and clearly does not directly correlate with corresponding damage levels observed in the HD sample. The scattering yield in the lower dose (LD) sample is seen to rise slowly in a regular manner with depth until near the EOR where there is a small but perceptible change in the rate of increase. This suggests that a discrete change in the damage concentration or type occurs at this depth similar to that observed at the higher fluence. A comparison of the aligned spectra from the two implanted samples surprisingly shows that the yield from the top $\sim 1.0 \mu \mathrm{m}$ is the same in both samples. Apparently, there is some critical level for damage in this region after which no further growth occurs even though substantial numbers of Frenkel defects are produced within the region during subsequent irradiation. Therefore, two distinct regions are observed: in the 
Vaity of the ions' EOR, damage increases monotonically with dose until the lattice turns anorplious, while ahead of this region damage growth increases initially with dose but saturates at a low level. The damage remains saturated until further growth of the buried amorphous latyer encroaches on the region. This is seen in Fig. 4, which compares aligned spectrat from three simples which were implanted with $1.25-\mathrm{MeV} \mathrm{Si}{ }^{+}$ions at slightly different doses. The fomation of a buried layer is clear in all samples. The damage (i.e., scattering yield) ahead of the buried layer is seen to remain constant, even though there the layer grows with increasing dose. The abrupt change in the scattering yield in the vicinity of the a-c interface in all samples indicates a sharp transition between the two phases and is consistent with a layer-by-layer growth mechanism for the a-layer.

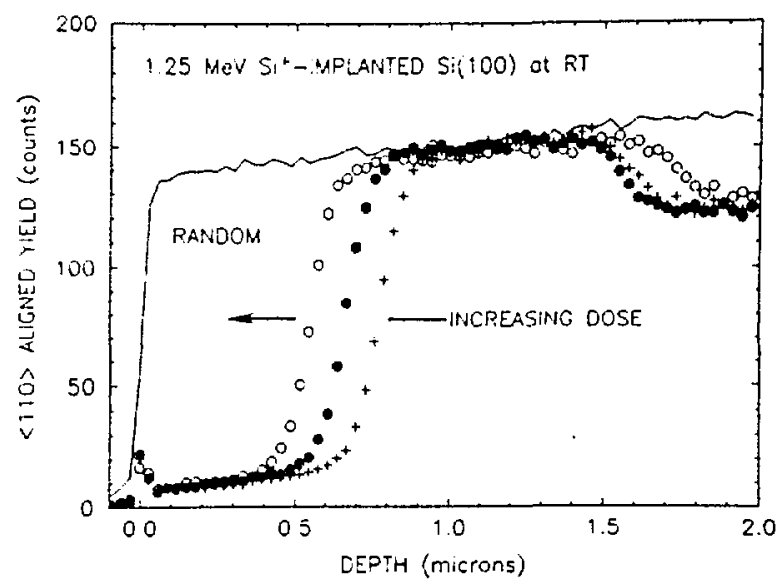

Fig. 4. Comparison of aligned spectra from Si single crystals implanted with different doses of 1.25-MeV Si+ ions.

Cross-sectional TEM micrographs from both the HD- and LD-implanted samples are presented in Fig. 5. The morphology determined from analysis of the micrographs is consistent with the RBS results above. The formation of the buried layer in the HD sample is clearly seen in Fig. 5(b). Extended defects and loops are evident both beyond the buried layer and in a narrow region ahead of the layer. However, the near-surface extending over a range of $\sim 1.0 \mu \mathrm{m}$ is remarkably free of any defects. It is clear that nucleation of extended defects such as dislocations is suppressed in this region. The absence of these types of defects plays an important role (which is discussed later) in the saturation of damage in this region. It is worth noting that the growth of the buried amorphous layer during continued irradiation occurs only at the a-c interface. The presence of dislocation loops in proximity to the a-c interface was characteristic in all samples examined in which an amorphous layer was formed. The morphology of the LDimplanted sample is shown in Fig. 5(a). No extended defects are seen over the top $\sim 1.0 \mu \mathrm{m}$, while a band of defects is located near the EOR region. These defects were stable to temperatures in excess of $600^{\circ} \mathrm{C}$ and are thought to be small dislocation loops.

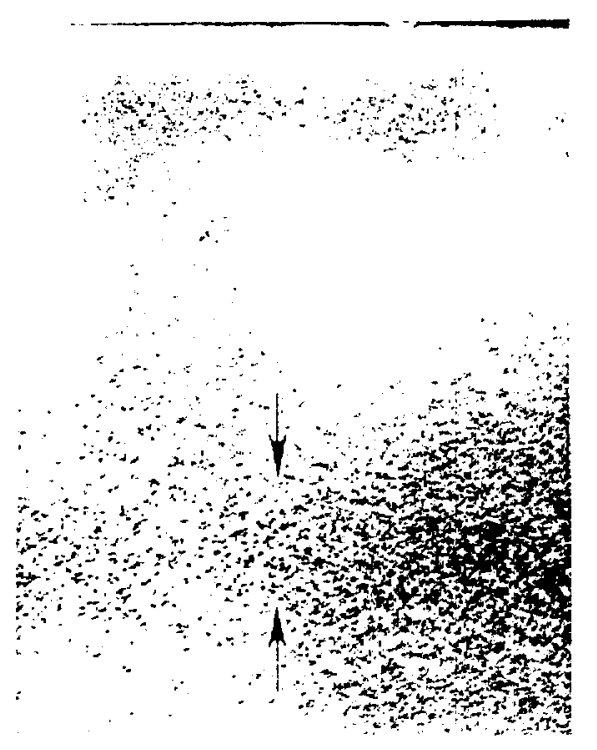

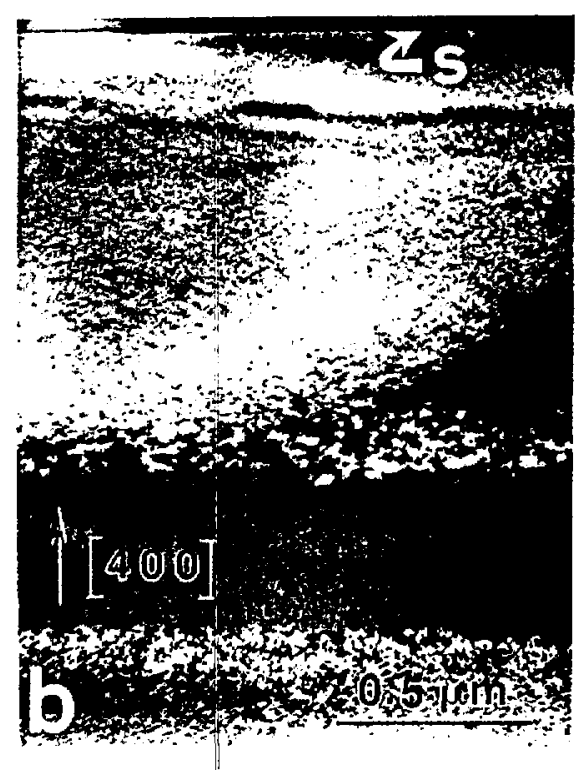

Fig. 5. Cross-sectional TEM micrograph from Si samples implanted with $1.25-\mathrm{MeV} \mathrm{Si}^{+}$ ions at a dose of (a) $0.25 \times 10^{\mathrm{i} 6}$ and $(\mathrm{b})$ $1.0 \times 10^{16} \mathrm{~cm}^{-2}$. 
- The absence of extended defects in the near-surface after high-energy ion-irradiation suggests that the damage determined by RBS consists of small defect clusters. Since defects anneal with a characteristic activation energy, they are often mobile in different time-temperature domains and can therefore be identified by their annealing behavior. Aligned spectra, before and after annealing at $200^{\circ} \mathrm{C}$ for $1 \mathrm{~h}$, from the HD Si${ }^{+}$-implanted sample are compared in Fig. 6 . This temperature is too low to anneal either dislocations or amorphous damage. It can be seen from the spectrat that, while no regrowth of the buried amorphous layer occurred during the anneal, most of the damage in tic near surface was removed. The characteristic annealing time (e.g., the time for annealing half of the damage) at $200^{\circ} \mathrm{C}$ for the defects in this region was $30 \mathrm{~min}$. This is similar to that for the divacancy which is known to form in Si under certain irradiation conditions 113]. Therefore, the dominant defect in the region where damage saturation occurs appears to be the divacancy.

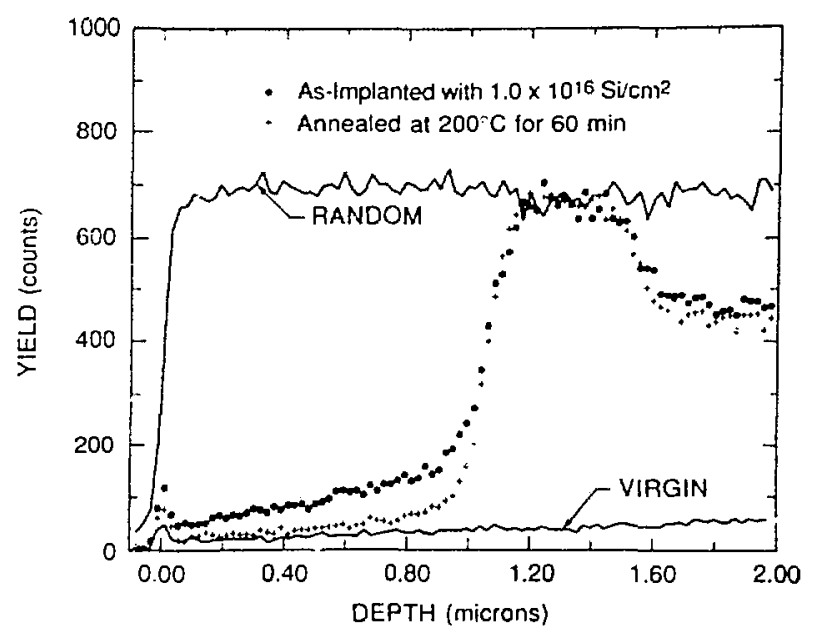

Fig. 6. Aligned spectra from a Si+ $\left(1.25 \mathrm{MeV}, 1.0 \times 10^{16} \mathrm{~cm}^{-2}\right)$ implanted Si sample before and after annealing at $200^{\circ} \mathrm{C}$ for $1 \mathrm{~h}$.

Observations presented to this point are thought to be the result of a homogeneous nucleation and growth mechanism for damage in Si. Insight into details of the model which account for damage saturation is gained by analysis of the defect reactions using chemical rate equations. Rather than starting from first principles, only those reactions which are consistent with observation will be allowed. This eliminates any reactions which lead to nucleation or growth of extended defects such as dislocations. The reason for the absence of these kinds of defects in the saturated region is unclear at this point. Reactions involving the Frenkel defects are therefore limited to (a) interstitial/vacancy recombination, (b) divacancy formation by vacancy pairing, and (c) divacancy annealing by combination with an interstitial. This reaction leads to the following rate equations for growth of the different defect populations:

$$
\begin{aligned}
& \frac{d n_{i}}{d t}=G-k_{1} n_{i} n_{v}-k_{2} n_{i} n_{o}, \\
& \frac{d n_{v}}{d t}=G-k_{1} n_{i} n_{v}-k_{3} n_{v}^{2}+k_{2} n_{i} n_{o}, \\
& \frac{d n_{o}}{d t}=\frac{1}{2} k_{3} n_{v}^{2}-k_{2} n_{i} n_{o},
\end{aligned}
$$

where $n_{i}, n_{v}, n_{O}$ are the respective concentration of the interstitial, vacancy and divacancy, and the k's are the reaction rate constants. The homogeneous generation rate of Frenkel defects by the ions is given by G. It is clear that there exists a steady-state solution of these nonlinear differential equations. This occurs as a result of a detailed balancing between the annihilation and creation rates for each type of defect. This balancing is responsible for the saturation of the damage and clearly would not be possible if unsaturable sinks, such as dislocations, were present in the reaction volume. A square ront dependence of the saturation level on dose rate is predicted by the steady-state solution. This dose-rate dependence was confirmed using different current 
dersity implants $\left(0.4\right.$ and $\left.0.1 \mu \mathrm{A} / \mathrm{cm}^{2}\right)$. However, the dependency was found to be slightly weaker than predicted, varying approximately as the cube root of the dose rate.

\section{Viedium-Energy $(100-\mathrm{keV}) \mathrm{Si}+$ Ion Irradiation}

Further investigations into damage effects which occur during high-energy ion irradiation were done using lower-energy $(100-\mathrm{keV})$ ions to predamage the near-surface region. The morphology of the damage formed during the $100-\mathrm{keV}$ ion irradiation depended on the ion fluence used. The subsequent interaction of high-energy ions with the predamage was then investigated. However, before this interaction could be studied it was necessary to characterize the nature of the damage produced by the $100-\mathrm{keV}$ ions and the growth behavior. Different annealing stages were studied to determine the nature of the damage present in the implanted region. Well defined annealing stages have been observed in ion-irradiated $\mathrm{Si}$ at 250 and $600^{\circ} \mathrm{C}$. As previously discussed, the lower temperature stage is associated with the divacancy defect, while $600^{\circ} \mathrm{C}$ is sufficiently high to remove amorphous damage [14]. It should be cautioned that the identification of the divacancy as the defect annealed at $250^{\circ} \mathrm{C}$ is somewhat restrictive. Higher-order vacancy complexes and simple defect clusters which act as trapping sites during the anneal can be involved in this stage. Extended defects (such as dislocations, staking faults, and twins) generally require temperatures significantly above $600^{\circ} \mathrm{C}$ to anneal. The distribution of damage was determined after each anneal stage from ion channeling spectra. The profiles could then be manipulated to yield the distribution of a particular defect. For example, the difference in the asimplanted damage and that remaining after $250^{\circ} \mathrm{C}$ annealing yields the divacancy profile.

The variation of the different defects with implantation dose in a depth range of $80-110 \mathrm{~nm}$ is shown in Fig. 7. This depth range brackets the EOR of the $100-\mathrm{keV}$ ions. Only the distribution of the divacancy (i.e., simple defect clusters) and amorphous damage are shown. While small dislocation loops were observed over a portion of the fluence range, their contribution to the total damage fraction was very small $(\sim 0.02)$. Total damage is seen to increase with dose in a very nonlinear manner; initially growing sublinearly with dose, but afterwards increasing quit rapidly. Such behavior for ion-implanted $\mathrm{Si}$ has been reported by others $[4,15]$. The transition between these two behaviors is seen to occur at a critical dose of $\sim 2 \times 10^{14} \mathrm{~cm}^{-2}$. The range of dose below this transition point will be referred to as region I, while region II will reference the range above. Not only does the behavior of the total damage change at this critical point but that of the different defects is also seen to diverge. The damage produced in region I consists almost entirely of the divacancy defect, while amorphous damage is dominant in region II. The critical dose marks the onset of the growth of amorphous damage and also a marked increase in the divacancy population which eventually disappears when the region is completely amorphized. The divergent behavior of both the total damage, and the divacancy component is seen to correlate with the onset of amorphous growth.

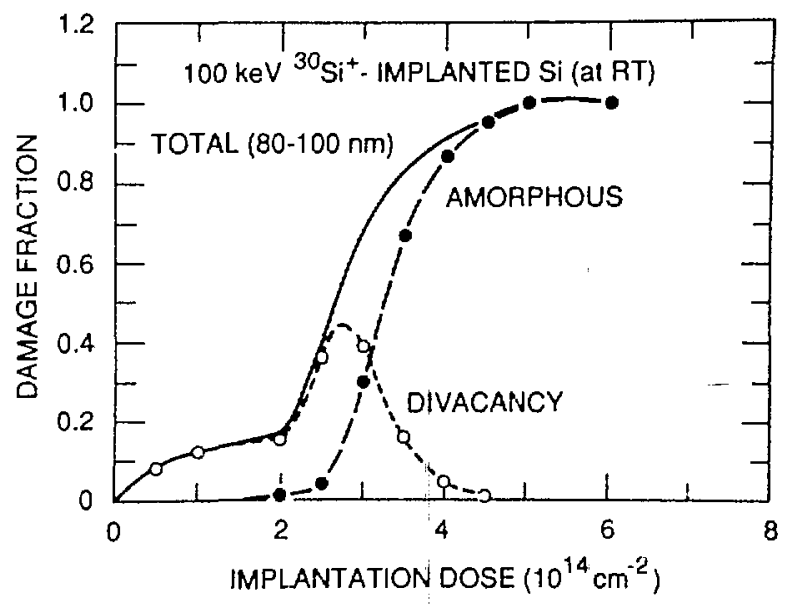

Fig. 7. Dose dependence of damage (and its components) produced during 100 -keV Si+ ion irradiation of $\mathrm{Si}(100)$ single crystal. 
. It is clear that different defect processes must dominate in the two growth regimes. During the initial sublinear regime, simple defects are formed and their growth greatly slows near the mansition. These defects (in region I) and their growth behavior is similar to that which occurs in the near-surface region ahead of the EOR in Si irradiated with the megaelectron volt ions. The constraned growth in the present case is therefore thought to be the result of a mechanism similar to that which produces damage saturation during MeV ion irradiation. It should be recalled that constrained growth was attributed to the detailed balancing between simple defect reactions in the irradiated volume.

The trinsition between the two growth regimes must coincide with some nucleation phenomena which precipitates the rapid growth of damage [4]. The presence of dislocation loops in both regions seems to discount their role in this process, although they may contribute to danage growth in region I. TEM showed that the loop size and density increased slightly through the transition between the two regions, but the change was continuous and seemed unrelated to the divergent behavior at the critical dose. Rather, the onset of amorphous damage growth at the critical point seemed to initiate the different growth behavior. It is believed that the formation of amorphous damage within the irradiated volume significantly alters the balance between the defect reactions which is established during the initial growth, as is evidenced by the rapid increase in the divacancy defect at the critical point. While the details of the process are not clear, the amorphous $\mathrm{Si}$ is thought to trap interstitial-type defects which in turn removes the constraint on the divacancy growth in the surrounding crystalline regions, permitting it to increase until it reaches the critical value for amorphization. Therefore, the ion-induced c-a phase transition in $S i$ is considered to be a cooperative process in which its onset triggers a rapid growth of other defect-types which then leads to further amorphization. It should be mentioned that this cooperative mechanism is consistent with the growth behavior of the buried amorphous layer which occurs during high-energy ion irradiation. The growth of the layer is recalled to occur only within the the vicinity of the a-c interface, while ahead of the layer damage remained saturated at a low level. The presence of the a-c interface appears to alter the balance between the various defect reactions in its vicinity leading to an unconstrained growth of damage which results in further amorphization.

Since damage nucleation during both medium- and high-energy ion irradiation has been assumed to be homogeneous in nature (rather than heterogeneous at the site of the collision cascade), it is of interest to compare the $100 \mathrm{keV}$ results to the predictions of the cascade model given by Eqn. (1). The curves given for different values of $m$ (the number of cascade overlaps required to produce the amorphous transition) are shown as the solid lines in the Fig. 8 . It is clear that, for heterogeneous growth, the amorphous transition becomes more abrupt with dose as the value of $\mathrm{m}$ increases. However, it is seen that the actual a-c transition which occurs in $\mathrm{Si}$ is more abrupt than that predicted by Eqn. (1), even for $m=12$. It therefore seems clear that heterogeneous nucleation does not dominate for self-ion implantation in Si.

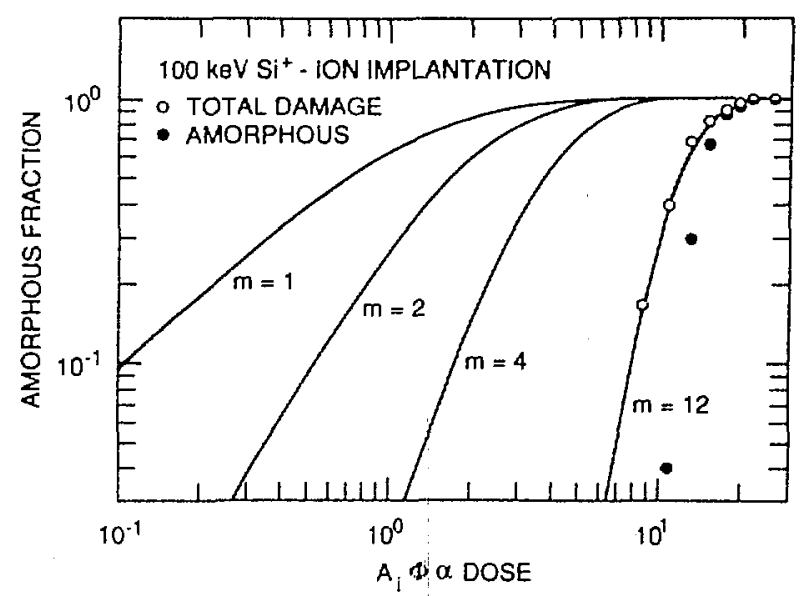

Fig. 8. The variation of amorphous damage with ion dose. Gibbon's model predictions are given for different values of $\mathrm{m}$ by the solid curves. Experimental values for both total and amorphous damaged produced by $100-\mathrm{keV} \mathrm{Si}^{+}$ions are given by the data points. 
The interaction of $\mathrm{MeV}$ ions with different predamage morphologies is illustrated in Fig. 9 . Aligned spectra in Figs. 9(a) and (b) are from samples predamaged with $2 \times 10^{14}$ and $3 \times 10^{14}$ $\mathrm{cm}^{-2}$ implants at $100 \mathrm{keV}$, respectively. A scattering peak is seen at $00.09 \mu \mathrm{m}$ in each of the (100 keV) as-implanted spectra indicating a peak in the damage distribution at this location. According to Fig. 7, the damage at the lower dose consists predominantly of simple defects (clusters), while substantial amounts of both amorphous damage and divacancy defects are present at the higher dose. The effect of high-energy ions on each of these different damage morphologies is seen in the figure by comparing the scattering yield in the as-implanted spectra (over a region extending to $\sim 0.3 \mu \mathrm{m}$ ) with that from samples subsequently irradiated with 1.25 $\mathrm{MeV} \mathrm{Si}+$ ions at a fluence of $10^{16} \mathrm{~cm}^{-2}$. It is immediately clear that the interaction of the highenergy ions with the two predamage morphologies results in strikingly different results. In Fig. 9 (a), it is seen that the predamage was annealed by high-energy irradiation, while in Fig. 9(b) substantial damage growth occurs as evidenced by the large scattering yield in the near-surface which approaches the amorpheus level. The large scattering yield from deep in the MeV ionirradiated samples is obviously the result of damage produced near the EOR of the 1.25-MeV Si+ ions. TEM examination of the samples confirmed the RBS results. Implantation with $2 \times 10^{14}$ $\mathrm{Si}^{+} / \mathrm{cm}^{2}$ at $100 \mathrm{keV}$ produces a band of dislocation loops centered near $\sim 0.09 \mu \mathrm{m}$ which were observed to be slightly coarsened after high-energy, ion irradiation. Therefore, the ion-induced annealing in this sample is thought to be the result of a reduction in the divacancy concentration.

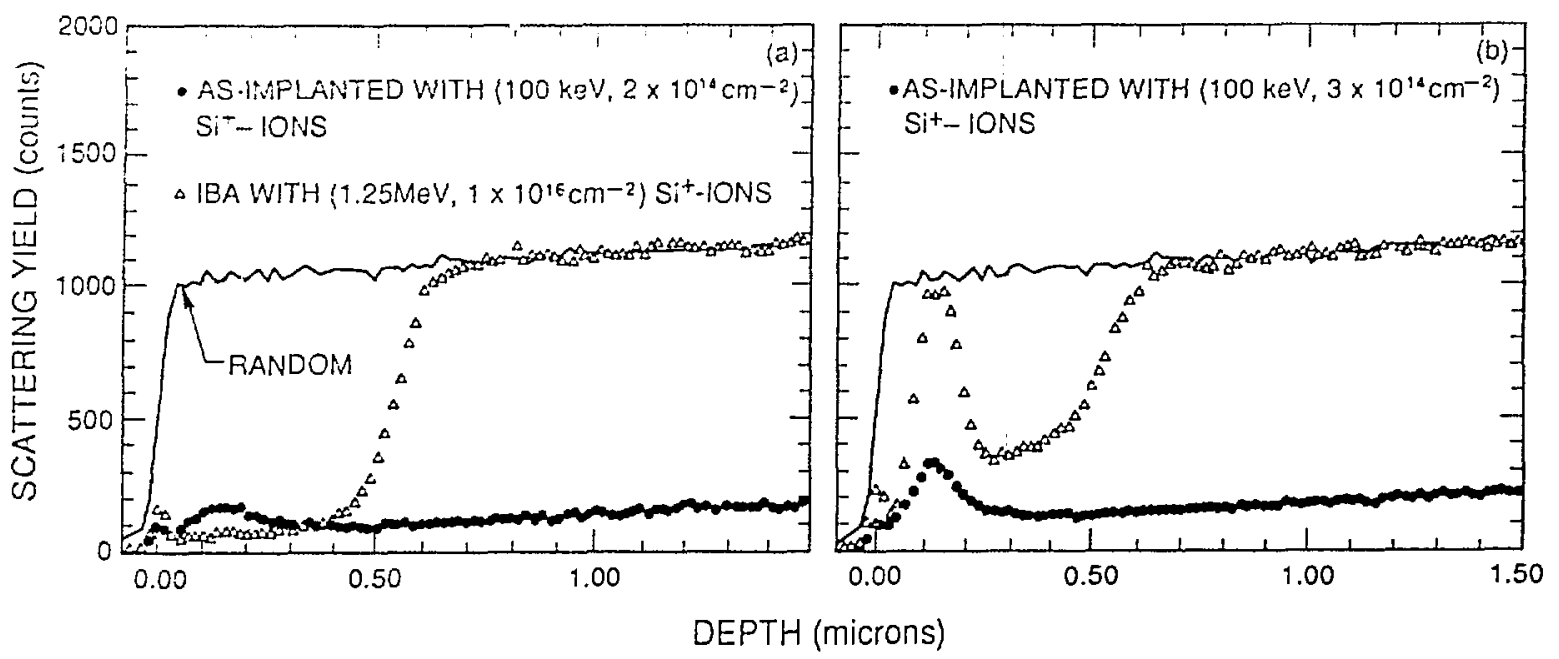

Fig. 9. Comparison of aligned spectra showing the effect of $1.25-\mathrm{MeV} \mathrm{Si}^{+}$ions on samples predamaged with $100-\mathrm{keV} \mathrm{Si}^{+}$ions at (a) $2 \times 10^{14}$ and (b) $3 \times 10^{14} \mathrm{~cm}^{-2}$.

At the higher fluence $\left(3 \times 10^{14} \mathrm{~cm}^{-2}\right)$, a band of small dislocation loops was also observed along with microdiffraction ring patterns consistent with amorphous Si. Damage growth which occurred during the subsequent $\mathrm{MeV}$ ion irradiation was found to result in the formation of a continuous, buried amorphous layer in the predamaged region. The markedly different nature of the interaction of the MeV ions on either side of the critical point (which marks the transition in the medium-energy, ion-induced damage growth) illustrates the importance of the defect morphology in this process. Predamaging at fluences below the critical point (in region I) leads to an interaction which results in substantial annealing of the damage, while above this value (in region II) rapid damage growth occurs. The reason for such behavior is readily discerned. Remember that, in the absence of any predamage, only divacancy defects are observed in the near-surface after the passage of $\mathrm{MeV}$ ions, and their concentration saturates during irradiation at a very low value. Predamaging in region I creates a very similar damage morphology, although the concentration of the divacancy defect is higher. This high concentration represents a supersaturation of these defects relative to the dynamical conditions established during high-energy ion irradiation and as such is expected to decrease during irradiation to a value consistent with it steady-state value. However, amorphous damage is formed over the entire region II. This type of damage has been shown to disrupt the the dynamical balancing which constrains damage growth. Therefore, it is not surprising to find that the interaction of the $\mathrm{MeV}$ ions with the amorphous damage results in rapid damage growth. 


\section{CONCLUSION}

The dose dependence of damage production during $100-\mathrm{keV}$ self-ion irradiation of Si was determined. Two distinct regimes were observed: an initial regime in which growth is constrained and only simple defects are formed, followed by a regime of rapid, unconstrained growth which results in complete amorphization of the lattice. Damage nucleation over an extended range of energy for self-ion irradiation of $\mathrm{Si}$ was shown to be dominated by a homogeneous mechanism. In this model, the rapid growth during 100-keV ion irradiation is precipitated by the onset of amorphization. This is envisioned to occur as a result of a cooperative mechanism iit which the formation of amorphous damage leads to further amorphization by promoting growth of other types of defects. The homogeneous model was also shown to account for the saturation of damage in the surface region which occurs during high-energy bombardment, as well as the layer-by-layer growth of the buried amorphous layer formed near the EOR of the ions. Markedly different responses were observed in predamaged Si during irradiation with high-energy ions, varying from damage annealing to rapid growth. These different responses were shown to be consistent with the damage growth model.

\section{ACKNOWLEDGMENTS}

Research was sponsored by the Division of Materials Sciences, U.S. Department of Energy under contract DE-AC05-84OR21400 with Martin Marietta Energy Systems, Inc.

\section{REFERENCES}

1. See Review by J. W. Corbett, J. P. Karins, and T. Y. Tan, Nucl. Instrum. and Methods 182/183, 457 (1981).

2. See Review by D. A. Thompson, Radiat. Eff. 56, 105 (1981).

3. L. Eriksson, J. A. Davies, N. G. E. Johansson, and J. W. Mayer, J. Appl. Phys. 40, 842 (1969).

4. L. T. Chadderton, Radiat. Eff. 8, 77 (1971).

5. J. A. Brinkman, J. Appl. Phys. 25, 961 (1954).

6. M. L. Swanson, J. R. Parsons, and C. W. Hoelke, Radiat. Eff. 9, 249 (1971).

7. L. M. Howe and M. H. Rainville, Nucl. Instrum. and Methods 182/183, 143 (1981).

8. J. Narayan, O. S. Oen, D. Fathy, and O. W. Holland, Mater. Lett. 3, 67 (1985).

9. M. O. Ruault, J. Chaumont, J. M. Penisson, and A. Bourret, Philos. Mag. 50, 667 (1984).

10. J. F. Gibbons, Proceedings of the IEEE 60, 1062 (1972).

11. J. R. Dennis and E. B. Hale, J. Appl. Phys. 49, 1119 (1978).

12. J. P. Biersack, Nucl. Instrum. and Methods 174, 257 (1980).

13. F. L. Vook and H. J. Stein, Radiat. Eff. 2, 23 (1969).

14. G. L. Olson, A. Kokorowski, J. A. Roth, and L. D. Hess, in Laser-Solid Interactions and Transient Thermal Processing of Materials, ed. by J. Narayan, W. L. Brown, and R. A. Lemons (North-Holland, New York, 1983) p. 141.

15. F. H. Eisen and B. Welch, Radiat. Eff. 7, 143 (1971). 